\title{
Evaluation of a High-Accuracy MacCormack- Type Scheme Using Benchmark Problems
}

R. Hixon

Institute for Computational Mechanics in Propulsion

Cleveland, Ohio

March 1997

Prepared for

Lewis Research Center

Under Cooperative Agreement NCC3-531

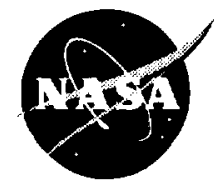

National Aeronautics and

Space Administration

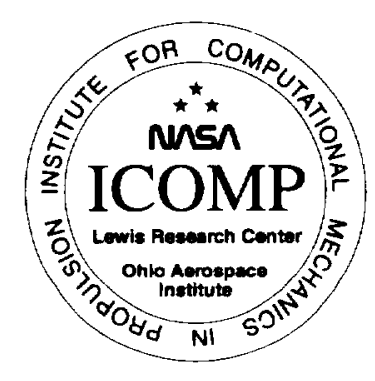




\title{
Evaluation of a High-Accuracy MacCormack-Type Scheme Using Benchmark Problems
}

\author{
R. Hixon \\ Institute for Computational Mechanics in Propulsion \\ NASA Lewis Research Center \\ Cleveland, $\mathrm{OH} 44135$
}

\begin{abstract}
Due to their inherent dissipation and stability, the MacCormack scheme and its variants have been widely used in the computation of unsteady flow and acoustic problems. However, these schemes require many points per wavelength in order to propagate waves with a reasonable amount of accuracy. In this work, the linear wave propagation characteristics of MacCormack-type schemes are shown by solving several of the CAA Benchmark Problems.
\end{abstract}

\section{Introduction}

In the field of computational aeroacoustics, numerical schemes are expected to propagate waves accurately for long distances over long periods of time. In order to accomplish this goal, a certain number of spatial points are required per wavelength to model each wave, and a certain time step is required in order to model the wave's movement in time. It is desirable from a computational standpoint to reduce the number of points required per wavelength and increase the size of the allowable time step.

One popular and well-tested method uses a modification of the MacCormack scheme ${ }^{1}$, which is second order accurate in time and fourth order accurate in space. This extension of the MacCormack scheme is known as the 2-4 scheme, and was described by Gottlieb and Turkel. ${ }^{2}$ This scheme has been used successfully on a wide range of fluid and 
aeroacoustics problems. ${ }^{3-15}$ Sankar, Reddy, and Hariharan have evaluated this scheme for aeroacoustics applications. ${ }^{16}$ It has been extended to sixth- order spatial accuracy by Bayliss, et. al. (2-6 scheme $)^{17}$, and an extension of the 2-4 scheme to fourth-order time accuracy is described by Viswanathan and Sankar. ${ }^{18}$

Building on this previous work, a new high-accuracy MacCormack-type scheme has been developed for use in computational aeroacoustics. ${ }^{19}$ This scheme has been successfully applied to the real-world problem of supersonic jet noise prediction. ${ }^{20}$ In this paper, the performance of this scheme will be evaluated using the benchmark problems of the first and second CAA workshops. The results are used to quantify the performance of the various schemes.

\section{Numerical Formulation}

In this work, four previously existing MacCormack-type schemes will be used: the classical MacCormack scheme, the 2-4 scheme of Gottlieb and Turkel, the 2-6 scheme of Bayliss, et. al., and the 4-4 scheme described by Viswanathan and Sankar.

In addition to these schemes, the high-accuracy MacCormack-type scheme of Hixon will be used. This scheme utilizes the Dispersion Relation Preserving methodology of Tam and $\mathrm{Webb}^{21}$ for the spatial discretization and the 4-6 Low-Dispersion and -Dissipation Runge-Kutta scheme of $\mathrm{Hu}$, et. al. ${ }^{22}$ for the time integration. It is formally fourth-order accurate in time and space for linear problems.

The time marching method used by these MacCormack-type schemes can be written as follows:

$$
\begin{aligned}
& \tilde{Q}^{(1)}=\tilde{Q}^{k} \\
& \tilde{Q}^{(2)}=\tilde{Q}^{k}+\alpha_{2} \Delta t F\left(\tilde{Q}^{(1)}\right) \\
& \tilde{Q}^{(3)}=\tilde{Q}^{k}+\alpha_{3} \Delta t F\left(\tilde{Q}^{(2)}\right)
\end{aligned}
$$




$$
\begin{aligned}
\tilde{Q}^{(4)} & =\tilde{Q}^{k}+\alpha_{4} \Delta t F\left(\tilde{Q}^{(3)}\right) \\
\tilde{Q}^{(5)} & =\tilde{Q}^{k}+\alpha_{5} \Delta t F\left(\tilde{Q}^{(4)}\right) \\
\tilde{Q}^{(6)} & =\tilde{Q}^{k}+\alpha_{6} \Delta t F\left(\tilde{Q}^{(5)}\right) \\
\tilde{Q}^{k+1}=\tilde{Q}^{k}+\Delta t & {\left[\begin{array}{l}
\beta_{1} F\left(\tilde{Q}^{(1)}\right)+\beta_{2} F\left(\tilde{Q}^{(2)}\right)+ \\
\beta_{3} F\left(\tilde{Q}^{(3)}\right)+\beta_{4} F\left(\tilde{Q}^{(4)}\right)+ \\
\beta_{5} F\left(\tilde{Q}^{(5)}\right)+\beta_{6} F\left(\tilde{Q}^{(6)}\right)
\end{array}\right] }
\end{aligned}
$$

where the values of the coefficients are given in Table 1. Notice that the 4-6 time marching method alternately uses four and six stages to move to the next time level. Each spatial derivative uses biased differencing, either forward or backward, providing inherent dissipation for the solver.

Using a spatial derivative at point $j$ as an example,

Forward:

$$
\left.\frac{\partial \tilde{Q}}{\partial r}\right|_{i}=\frac{1}{\Delta r}\left(\begin{array}{l}
a_{-1} \tilde{Q}_{i-1}+a_{0} \tilde{Q}_{i} \\
+a_{1} \tilde{Q}_{i+1}+a_{2} \tilde{Q}_{i+2} \\
+a_{3} \tilde{Q}_{i+3}
\end{array}\right)^{k}
$$

Backward:

$$
\left.\frac{\partial \tilde{Q}}{\partial r}\right|_{i}=\frac{-1}{\Delta r}\left(\begin{array}{l}
a_{3} \tilde{Q}_{i-3}+a_{2} \tilde{Q}_{i-2} \\
+a_{1} \tilde{Q}_{i-1}+a_{0} \tilde{Q}_{i} \\
+a_{-1} \tilde{Q}_{i+1}
\end{array}\right)^{k}
$$

The sweep directions are reversed between each stage of the time marching scheme to avoid biasing, and the first sweep direction in each time step is alternated as well. This gives a four-step time marching cycle (using the 4-6 time marching method to illustrate): 


\begin{tabular}{|c|c|c|c|c|}
\hline & 2nd order & 4th order & Hu 4-6 Step1 & Hu 4-6 Step2 \\
\hline$\alpha_{2}$ & 1 & $1 / 2$ & $1 / 2$ & 0.353323 \\
\hline$\alpha_{3}$ & 0 & $1 / 2$ & $1 / 2$ & 0.999597 \\
\hline$\alpha_{4}$ & 0 & 1 & 1 & 0.152188 \\
\hline$\alpha_{5}$ & 0 & 0 & 0 & 0.534216 \\
\hline$\alpha_{6}$ & 0 & 0 & 0 & 0.603907 \\
\hline$\beta_{1}$ & $1 / 2$ & $1 / 6$ & $1 / 6$ & 0.0467621 \\
\hline$\beta_{2}$ & $1 / 2$ & $1 / 3$ & $1 / 3$ & 0.137286 \\
\hline$\beta_{3}$ & 0 & $1 / 3$ & $1 / 3$ & 0.170975 \\
\hline$\beta_{4}$ & 0 & $1 / 6$ & $1 / 6$ & 0.197572 \\
\hline$\beta_{5}$ & 0 & 0 & 0 & 0.282263 \\
\hline$\beta_{6}$ & 0 & 0 & 0 & 0.165142 \\
\hline
\end{tabular}

Table I: Coefficients for Runge-Kutta Time Stepping Schemes.

\begin{tabular}{|c|c|c|c|c|c|}
\hline & $a_{-1}$ & $a_{0}$ & $a_{1}$ & $a_{2}$ & $a_{3}$ \\
\hline 2nd order [1] & 0 & $\frac{-1}{\Delta x}$ & $\frac{1}{\Delta x}$ & 0 & 0 \\
\hline 4th order [2] & 0 & $\frac{-7}{6 \Delta x}$ & $\frac{8}{6 \Delta x}$ & $\frac{-1}{6 \Delta x}$ & 0 \\
\hline $\begin{array}{c}\text { 6th order } \\
{[17]}\end{array}$ & 0 & $\frac{-37}{30 \Delta x}$ & $\frac{45}{30 \Delta x}$ & $\frac{-9}{30 \Delta x}$ & $\frac{1}{30 \Delta x}$ \\
\hline DRP/opt & $\frac{-0.30874}{\Delta x}$ & $\frac{-0.6326}{\Delta x}$ & $\frac{1.2330}{\Delta x}$ & $\frac{-0.3334}{\Delta x}$ & $\frac{0.04168}{\Delta x}$ \\
\hline
\end{tabular}

Table II: Coefficients for MacCormack-Type Schemes 


$$
\begin{aligned}
& Q^{k+1}=L_{B F B F} Q^{k} \\
& Q^{k+2}=L_{F B F B F B} Q^{k+1} \\
& Q^{k+3}=L_{F B F B} Q^{k+2} \\
& Q^{k+4}=L_{B F B F B F} Q^{k+3}
\end{aligned}
$$

At the computational boundaries, flux quantities outside the boundaries are needed to compute the spatial derivatives; these fluxes are determined using third-order extrapolation from the interior nodes.

\section{Benchmark Problems}

These schemes are compared using linear benchmark problems from the first ${ }^{23}$ and second CAA Workshops.

\section{1) One Dimensional Problems}

Problem 1 of the first workshop requests the solution at $t=400$ of:

$$
u_{t}+u_{x}=0
$$

where

$$
\begin{aligned}
& u(0)=\frac{1}{2} \exp \left(-\ln (2)\left(\frac{x}{3}\right)^{2}\right) \\
& -20 \leq x \leq 450 \\
& \Delta x=1.0
\end{aligned}
$$

Problem 2 of the first workshop requests the solution at $t=400$ of:

$$
u_{t}+u_{r}+\frac{u}{r}=0
$$

where 


$$
\begin{aligned}
& u(r, 0)=0 \\
& u(5, t)=\sin (\omega t) \\
& \omega=\frac{\pi}{3}, \frac{\pi}{4}, \frac{\pi}{6} \\
& 5 \leq r \leq 450 \\
& \Delta r=1.0
\end{aligned}
$$

These problems test the ability of the scheme to accurately propagate linear waves of varying wavelengths for long distances of travel.

\section{2) Two Dimensional Problems}

The problems given for the second CAA Workshop are much more difficult. In this problem set, a 2-D circular cylinder of radius 0.5 is placed at the origin, and acoustic waves reflect and scatter from this curved surface. The governing equations are the twodimensional linearized Euler equations in polar coordinates:

$$
\left\{\begin{array}{l}
v_{r}^{\prime} \\
v_{\theta}^{\prime} \\
p^{\prime}
\end{array}\right\}_{t}+\left\{\begin{array}{c}
p^{\prime} \\
0 \\
v_{r}^{\prime}
\end{array}\right\}+\frac{1}{r}\left\{\begin{array}{c}
0 \\
p^{\prime} \\
v_{\theta}^{\prime}
\end{array}\right\}_{\theta}+\frac{1}{r}\left\{\begin{array}{c}
0 \\
0 \\
v_{r}^{\prime}
\end{array}\right\}=S
$$

For Problem 1, $S$ is a simple harmonic source at $r=4, \theta=0$, given by:

$$
S=\left\{\begin{array}{c}
0 \\
0 \\
\exp \left(-\ln (2) \frac{(x-4)^{2}+y^{2}}{(0.2)^{2}}\right) \sin (8 \pi t)
\end{array}\right\}
$$

The problem requests $\mathrm{rp}^{2}$ in the limit as $\mathrm{r}>>$ in the arc $180>\theta>90$.

In Problem 2, $S$ is an initial disturbance at time $t=0$, given by: 


$$
\left.S\right|_{t=0}=\left\{\begin{array}{c}
0 \\
0 \\
\exp \left(-\ln (2) \frac{(x-4)^{2}+y^{2}}{(0.2)^{2}}\right)
\end{array}\right\}
$$

For this problem, the pressure time history from $6<\mathrm{t}<10$ at three points is requested. These points are at $\theta=90^{\circ}$ (point $\mathrm{A}$ ), $\theta=135^{\circ}$ (point $\mathrm{B}$ ), and $\theta=180^{\circ}$ (point $\mathrm{C}$ ) at a radial distance of 5 from the origin.

\section{Boundary Conditions for Two-Dimensional Problems}

There are three boundary conditions which are used. At the cylinder surface $(r=0.5)$, the Thompson solid wall boundary condition is used, and the equations become:

$$
\left\{\begin{array}{l}
v_{r}^{\prime} \\
v_{\theta}^{\prime} \\
p^{\prime}
\end{array}\right\}_{t}+\left\{\begin{array}{c}
0 \\
0 \\
v_{r}^{\prime}-p^{\prime}
\end{array}\right\}_{r}+\frac{1}{r}\left\{\begin{array}{c}
0 \\
p^{\prime} \\
v_{\theta}^{\prime}
\end{array}\right\}_{\theta}+\frac{1}{r}\left\{\begin{array}{c}
0 \\
0 \\
v_{r}^{\prime}
\end{array}\right\}=S
$$

In this computation, three ghost points are used inside the surface for the radial derivative; their values are set as:

$$
\left\{\begin{array}{l}
v_{r}^{\prime} \\
v_{\theta}^{\prime} \\
p^{\prime}
\end{array}\right\}_{1-j}=\left\{\begin{array}{c}
-v_{r}^{\prime} \\
0 \\
p^{\prime}
\end{array}\right\}_{1+j}
$$

In the far field ( $r=R_{\max }$ ), the acoustic radiation condition is used:

$$
\left\{\begin{array}{c}
v_{r}^{\prime} \\
v_{\theta}^{\prime} \\
p^{\prime}
\end{array}\right\}+\left\{\begin{array}{c}
v_{r}^{\prime} \\
0 \\
p^{\prime}
\end{array}\right\}+\frac{1}{2 r}\left\{\begin{array}{c}
v_{r}^{\prime} \\
0 \\
p^{\prime}
\end{array}\right\}=S
$$

For the radial derivative at the outer boundary, three ghost points are used. The values of the variables at these ghost points are determined using third-order extrapolation from the interior values. 
At the symmetry planes $(\theta=0$ and $\theta=\pi)$, a symmetry condition is used. For example, around $\mathrm{i}=1$ :

$$
\left\{\begin{array}{l}
v_{r}^{\prime} \\
v_{\theta}^{\prime} \\
p^{\prime}
\end{array}\right\}_{1-i}=\left\{\begin{array}{c}
v_{r}^{\prime} \\
-v_{\theta}^{\prime} \\
p^{\prime}
\end{array}\right\}_{1+i}
$$

where $\mathrm{i}$ is the index in the azimuthal direction.

\section{Computational Grid for Two-Dimensional Problems}

For Problem 1, a 801 (radial) x 501 (azimuthal) grid was used, covering a domain of $0.5 \leq$ $\mathrm{r} \leq 20.5$ in the radial direction, and $0 \leq \theta \leq \pi$. Since the wavelength of the disturbance is 0.25 , this grid results in 7-10 points per wavelength. The exact results were given at the $r=$ 15 line, giving a maximum of 76 wavelengths of travel at $\theta=\pi$.

For Problem 2, a 201 (radial) x 301 (azimuthal) grid was used, covering a domain of $0.5 \leq$ $\mathrm{r} \leq 10.5$ in the radial direction, and $0 \leq \theta \leq \pi$. Since the transient problem only requires data from $6 \leq t \leq 10$, the outer radial boundary only has to be far enough away such that no reflections can reach any of the three data points during this time period.

\section{Results}

\section{1) One Dimensional Results}

The solutions of Benchmark Problem 1 at $t=400$ as calculated by the various schemes are shown in Figures 1-5. In this problem, a Gaussian pulse propagates in time and space for a given time, at which point the results are compared. On each figure, the solution is shown for various time steps to illustrate the effect of the time step on the accuracy of the time integration.

Figure 1 shows the solution obtained using the classical MacCormack scheme. Two

points are evident: first, the classical scheme is a perfect propagator at a CFL number of 
1.0 ; second, the scheme is very dissipative and dispersive for other time steps. Since perfectly uniform grids are unusual in real-world problems, the perfect propagation is not overly useful.

Figure 2 shows the solution obtained using the 2-4 scheme of Gottlieb and Turkel with time steps of $0.25,0.4$, and 0.5 . The dissipative nature of the scheme is evident, and the time step has a large effect on the dispersion error.

Figure 3 shows the solution obtained by the 2-6 method of Bayliss, et. al. Again, the solution shows dissipation and dispersion, with higher dispersion errors than the 2-4 scheme.

Figure 4 shows the solution obtained by the $4-4$ method described by Viswanathan and Sankar. The effect of the increased time accuracy on the dispersion error is immediately apparent. Another point of interest is that the four-stage, fourth-order Runge-Kutta scheme is dissipative at larger time steps.

Figure 5 shows the same time-stepping scheme using the optimized DRP spatial differencing. The solution obtained is very accurate and is also insensitive to the time step chosen.

Figures 6-8 compare the results of the 2-4 scheme to those of the optimized DRP scheme for Benchmark Problem 2. In this problem, a single-frequency spherical wave propagates outward from an impulsively-started vibrating sphere. As given, the problem has two parts: the first has 8 points per wavelength, and the second has six points per wavelength. To give the 2-4 scheme some chance of obtaining reasonable results, a third part was added which has 12 points per wavelength.

Figure 6 shows the results for 12 points per wavelength at 33.33 wavelengths of travel. The solution given by $2-4$ scheme shows dissipation error and some dispersion error, 
while the solution given by the optimized DRP scheme shows very little dispersion or dissipation error, even with a time step five times larger than that taken by the 2-4 scheme.

Figure 7 shows the results for 8 points per wavelength at 50 wavelengths of travel. The solution given by the $2-4$ scheme is very dissipated and shows some dispersion error. The solution given by the optimized DRP scheme shows some dispersion and dissipation error, but is still very good.

Figure 8 shows the results for 6 points per wavelength at 66.67 wavelengths of travel. Except for the initial transient, the wave has been completely damped by the $2-4$ scheme, while the optimized DRP scheme is still showing good accuracy in dispersion. However the wave has dissipated to approximately $65 \%$ of the exact value.

\section{2) Two Dimensional Results}

Results for Problem 1 of the second CAA Workshop are given in Figure 9. In order to avoid problems with the very large initial transient, a polynomial function was used to smoothly increase the amplitude of the forcing function. The time step used was limited by the stability of the solid wall boundary; for these calculations a time step of $\Delta t=$ 0.00245 was used $(C F L=0.786)$. The calculation was run to a time of 32.09 , with results being taken from $31.59 \leq \mathrm{t} \leq 32.09$. This calculation took a total of 6.27 hours of CPU time on a Cray Y/MP, running at 191 Mflops. The results are given at $r=15 \mathrm{D}$, and compare very well with the exact solution.

Results for Problem 2 are given in Figures 10-12. The results agree very well with the exact solution. This calculation, using a $\Delta \mathrm{t}$ of 0.0025 in order to print out the required results, took a total of $469 \mathrm{CPU}$ seconds on a Cray Y/MP, running at 175.5 Mflops. However, the code could run stably at a CFL number of $0.864(\Delta t=0.0045)$, requiring 
261 CPU seconds. With more stable solid wall boundary conditions, it is expected that the scheme can recover the $\mathrm{CFL}=1.4$ time step that has been seen previously.

Grid refinement studies were conducted for Problem 2; the effect of halving and doubling the grid are shown for Point $C$ in Figures 13 and 14. Point $C$ was chosen because it was the most distant point from the initial location of the pulse. In Figure 13, three computed results are shown: a half grid (101 x 151), the grid used $(201 \times 301)$, and a doubled grid (401 x 601). The two denser grids have nearly identical results, and compare very well with the exact solution. The coarsest grid, however, shows leading and trailing waves, some traveling much faster than the physical wave. This is due to the low resolution of the grid causing the solver to incorrectly allow high-frequency waves to travel faster than the speed of sound.

Figure 14 shows the transient peak at point C. The effect of increased grid is illustrated in this graph; the transient peak becomes closer and closer to the exact solution as the grid becomes denser. At this extreme amplification, it can be seen that the transient peak velocity is very slightly off with the grid used, but the answer is well within expected tolerances for this case.

\section{Conclusions}

In this work, the dispersive and dissipative characteristics of a new high-accuracy MacCormack-type scheme were investigated using benchmark problems of the first and second CAA workshops. The results show that this new scheme is very promising for computational aeroacoustics applications, requiring only 6-8 points per wavelength and allowing large time steps. 
The MacCormack-type schemes are of great interest due to their ease of programming and use, and inherent numerical dissipation. This work shows that this type of scheme can be optimized to perform very well.

\section{Acknowledgments}

This work was performed under grant NCC3-483 from the NASA Lewis Research Center. Dr. L. A. Povinelli was the Technical Monitor.

\section{References}

1) MacCormack, R. W., 'Lecture Notes in Physics', Vol. 8, Springer-Verlag, New York/Berlin, 1971, p. 151.

2) Gottleib, D. and Turkel, E., 'Dissipative Two-Four Method for Time Dependent Problems', Mathematics of Computation, Vol. 30, No. 136, 1976, pp. 703-723.

3) Bayliss, A. and Maestrello, L., 'Simulation of Instabilities and Sound Radiation in a Jet', AIAA Journal, Vol. 19, 1981, p. 835-841.

4) Maestrello, L., Bayliss, A., and Turkel, E., 'On the Interaction of a Sound Pulse with the Shear Layer of an Axisymmetric Jet', J. Sound and Vib., Vol. 74, 1981, p. 281301.

5) Bayliss, A. and Maestrello, L., 'On the Interaction of a Sound Pulse with the Shear Layer of an Axisymmetric Jet II: Heated Jets', J. Sound and Vib., Vol. 86, 1982, p. 395-409.

6) Maestrello, L. and Bayliss, A., 'Flowfield and Far Field Acoustic Amplification Properties of Heated and Unheated Jets', AIAA Journal, Vol. 20, 1982, p. 15391546. 
7) Bayliss, A., Maestrello, L., and Turkel, E., 'On the Interaction of a Sound Pulse with the Shear Layer of an Axisymmetric Jet III: Non-Linear Effects', J. Sound and Vib., Vol. 107, 1986, p. 167-175.

8) Mankbadi, R. R., Hayder, M. E., and Povinelli, L. A., 'Structure of Supersonic Jet Flow and Its Radiated Sound', AIAA Journal , Vol. 32, No. 5, May 1994, p. $897-$ 906.

9) Mankbadi, R. R., Shih, S.-H., Hixon, R., and Povinelli, L. A., 'Direct Computation of Acoustic and Flow Field of a Supersonic Jet Using Large-Eddy Simulation', AIAA Paper 95-0680, Reno, NV, Jan. 1995.

10) Shih, S.-H., Hixon, R., and Mankbadi, R. R., 'A Zonal Approach for Prediction of Jet Noise', CEAS/AIAA Paper 95-144, Munich, Germany, June 1995.

11) Mankbadi, R. R., Hixon, R., Shih, S.-H., and Povinelli, L. A., 'On the Use of Linearized Euler Equations in the Prediction of Jet Noise', AIAA Paper 95-0505, Reno, NV, Jan. 1995.

12) Hixon, R., Shih, S.-H., and Mankbadi, R. R., 'Direct Prediction of the ThreeDimensional Acoustic Field of a Supersonic Jet Using Linearized Euler Equations', CEAS/AIAA-95-116, June 1995.

13) Hixon, R., Shih, S.-H., and Mankbadi, R. R., 'Numerical Simulation of the Effect of Heating on Jet Noise', FED-Vol. 219, Computational Aeroacoustics, ASME 1995, p. 89-94.

14) Hixon, R., Shih, S.-H., and Mankbadi, R. R., 'Evaluation of Boundary Conditions for Computational Aeroacoustics', AIAA Journal, Oct. 1995. 
15) Hixon, R., Shih, S.-H., and Mankbadi, R. R., 'Effect of Inflow Disturbance on Linearized Euler Equation Prediction of Jet Noise', AIAA Paper 96-0752, Jan. 1996.

16) Sankar, L. N., Reddy, N. N., and Hariharan, N., 'A Comparative Study of Numerical Schemes for Aero-Acoustic Applications', FED-Vol. 147, Computational Aeroand Hydro-Acoustics, ASME 1993, p. 35-40.

17) Bayliss, A., Parikh, P., Maestrello, L., and Turkel, E., 'Fourth Order Scheme for the Unsteady Compressible Navier-Stokes Equation', ICASE Report 85-44, Oct. 1985.

18) Viswanathan, K. and Sankar, L. N., 'A Comparative Study of Upwind and MacCormack Schemes for CAA Benchmark Problems', ICASE/LaRC Workshop on Benchmark Problems in Computational Aeroacoustics, NASA CP 3300, Hampton, VA., May 1995, p. 185-195.

19) Hixon, R. 'On Increasing the Accuracy of MacCormack Schemes for Aeroacoustic Applications', AIAA Paper 97-1586, May 1997.

20) Hixon, R., Shih, S.-H., and Mankbadi, R. R., 'Effect of Coannular Flow on Linearized Euler Equation Predictions of Jet Noise', AIAA Paper 97-0284, Reno, NV, Jan. 1997.

21) Tam, C. K. W., and Webb, J, C., 'Dispersion-Relation-Preserving Finite Difference Schemes for Computational Acoustics', J. Comp. Physics, Vol. 107, 1993, p. 262281. 
22) Hu, F. Q., Hussaini, M. Y., and Manthey, J., 'Low-Dissipation and -Dispersion Runge-Kutta Schemes for Computational Acoustics', ICASE Report 94-102, Dec. 1994.

23) Tam, C. K. W., 'Benchmark Problems and Solutions', ICASE/LaRC Workshop on Benchmark Problems in Computational Aeroacoustics, NASA CP 3300 , Hampton, VA., May 1995, p. 1-13.

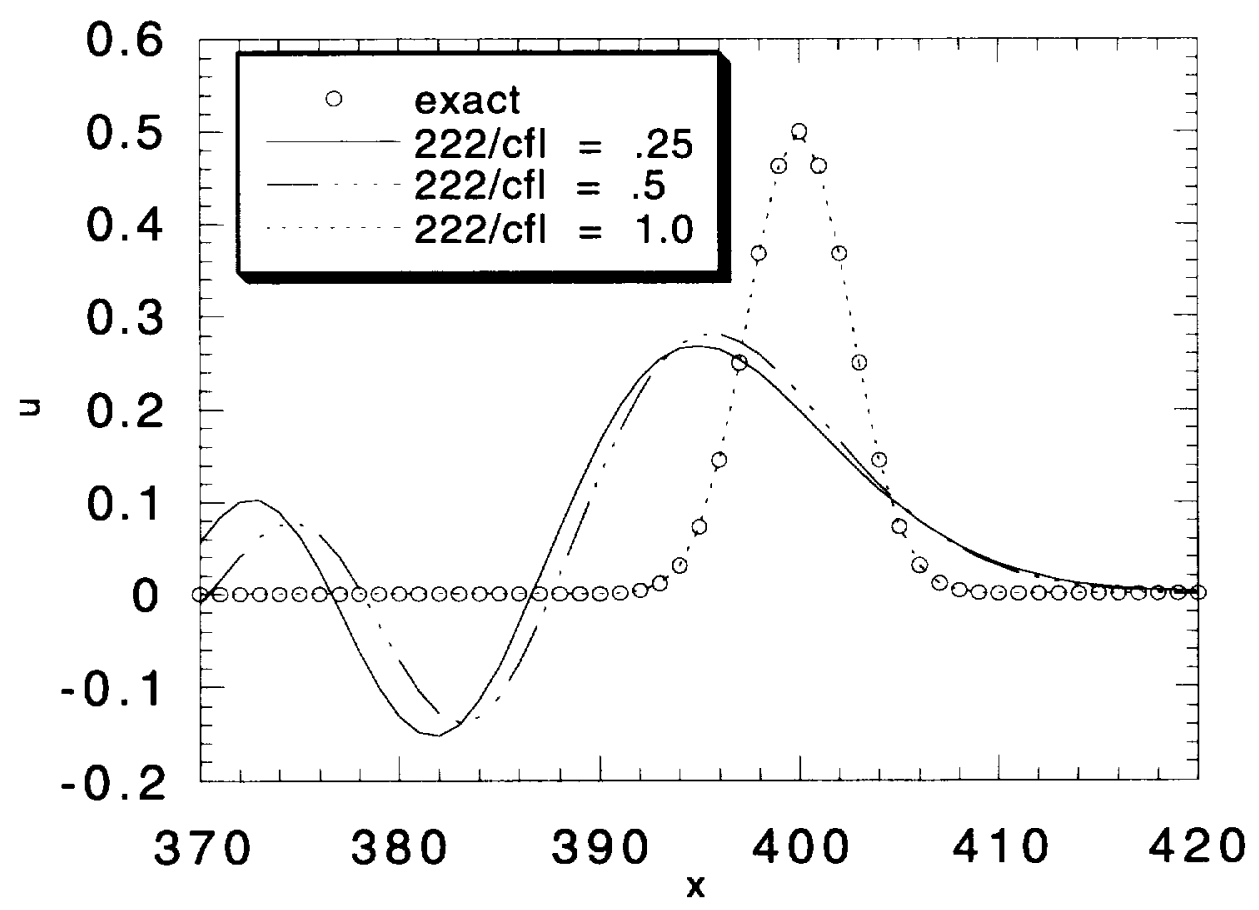

Figure 1.-Solution of Benchmark Problem 1 of the First CAA Workshop using Classical MacCormack Scheme. 


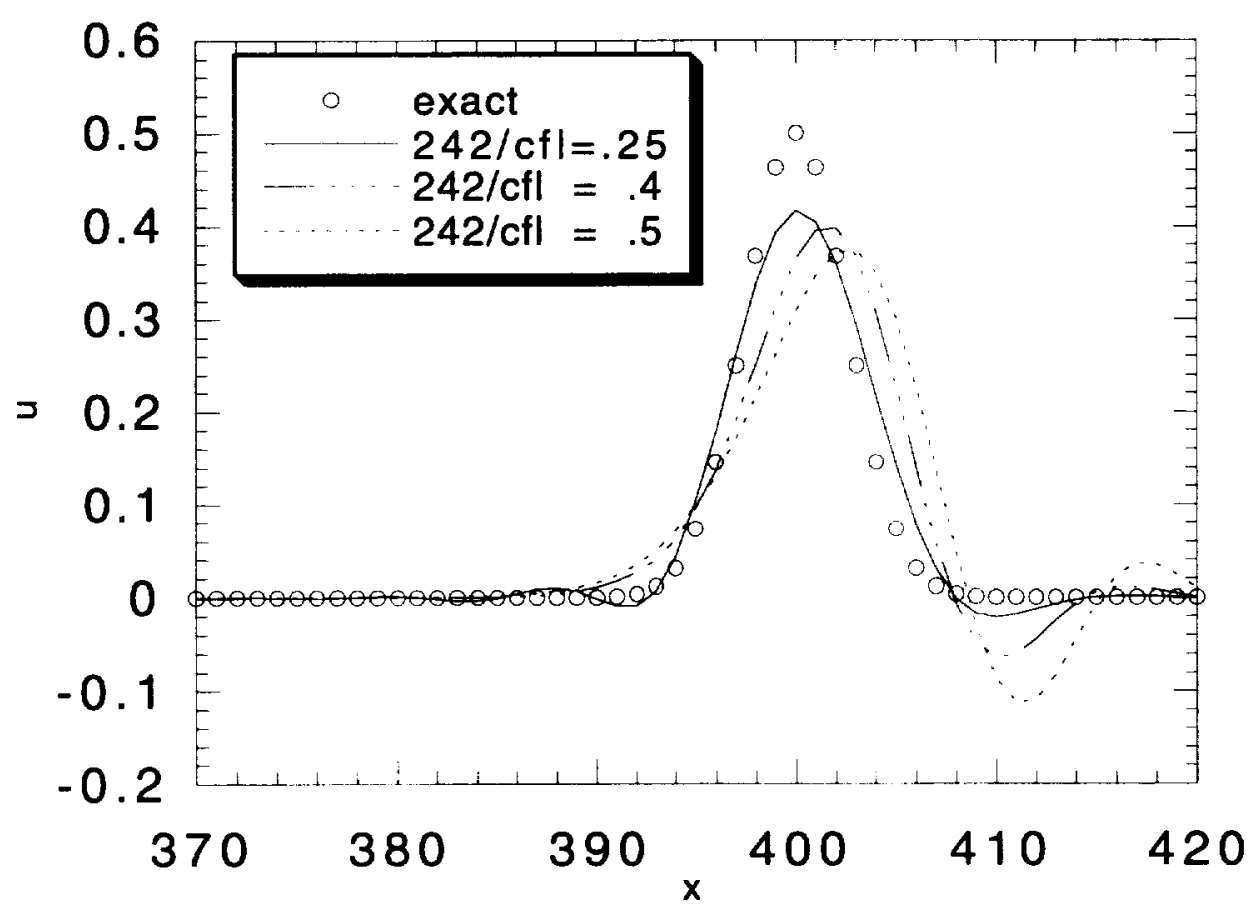

Figure 2.- Solution of Benchmark Problem 1 of the First CAA Workshop using 2-4 Scheme.

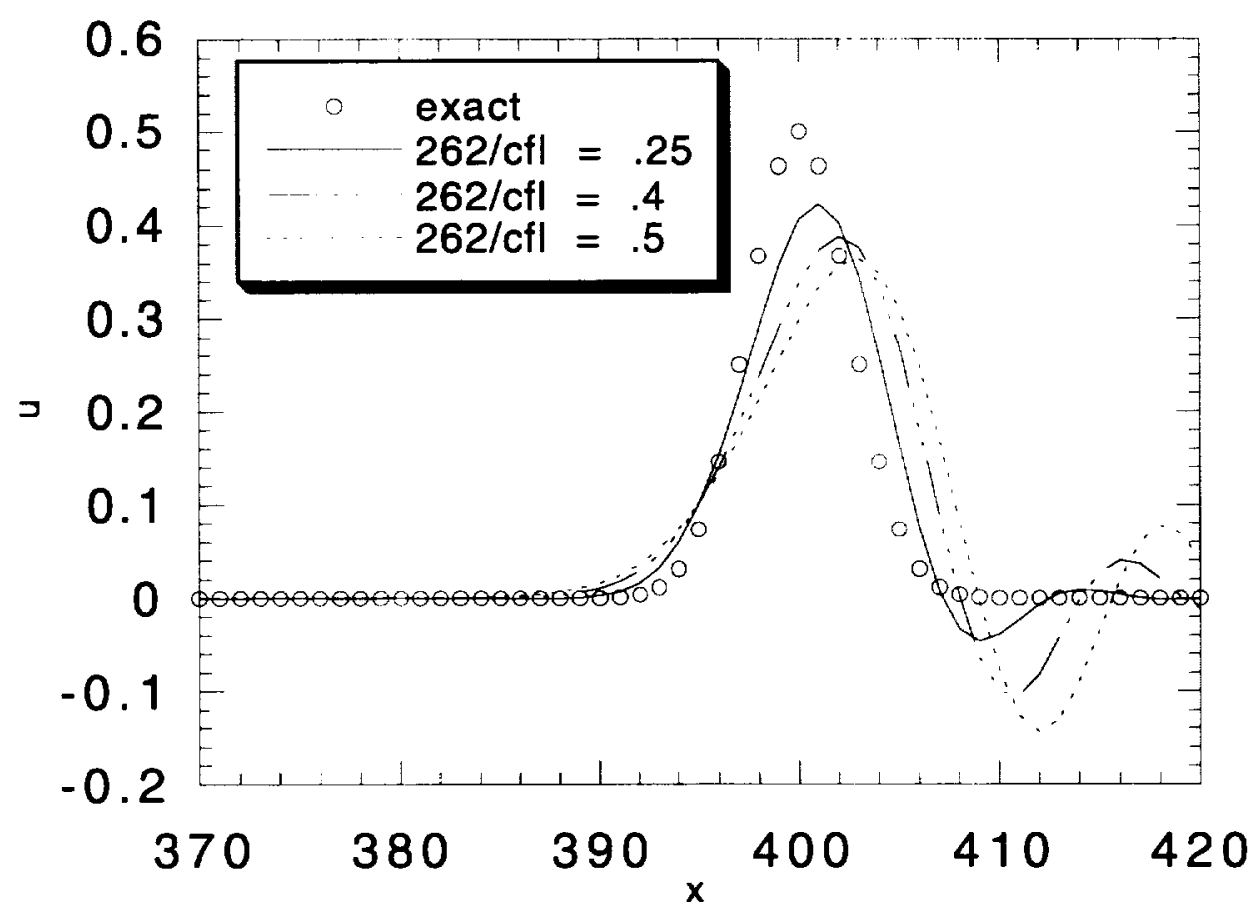

Figure 3.-Solution of Benchmark Problem 1 of the First CAA Workshop using 2-6 Scheme. 


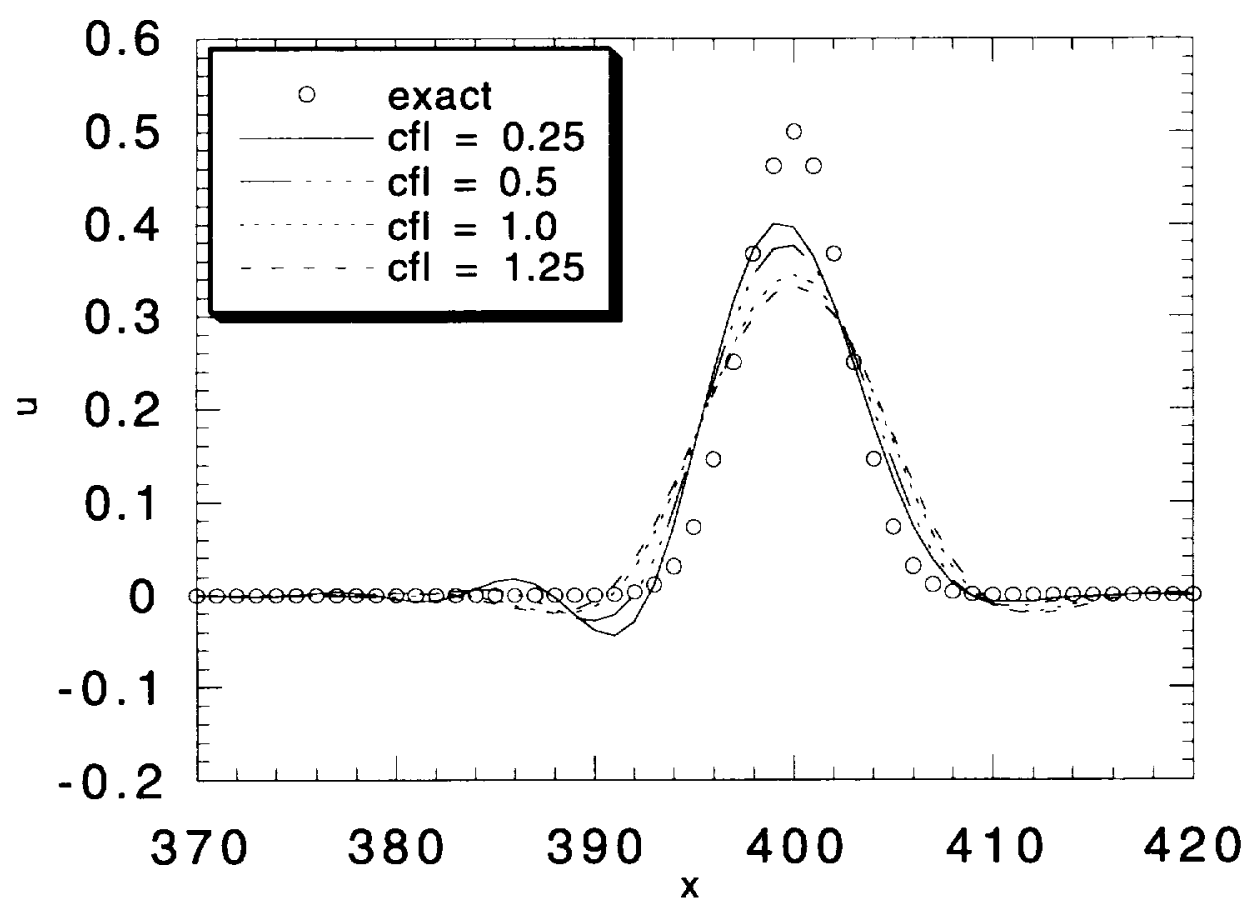

Figure 4.-Solution of Benchmark Problem 1 of the First CAA Workshop using 4-4 Scheme.

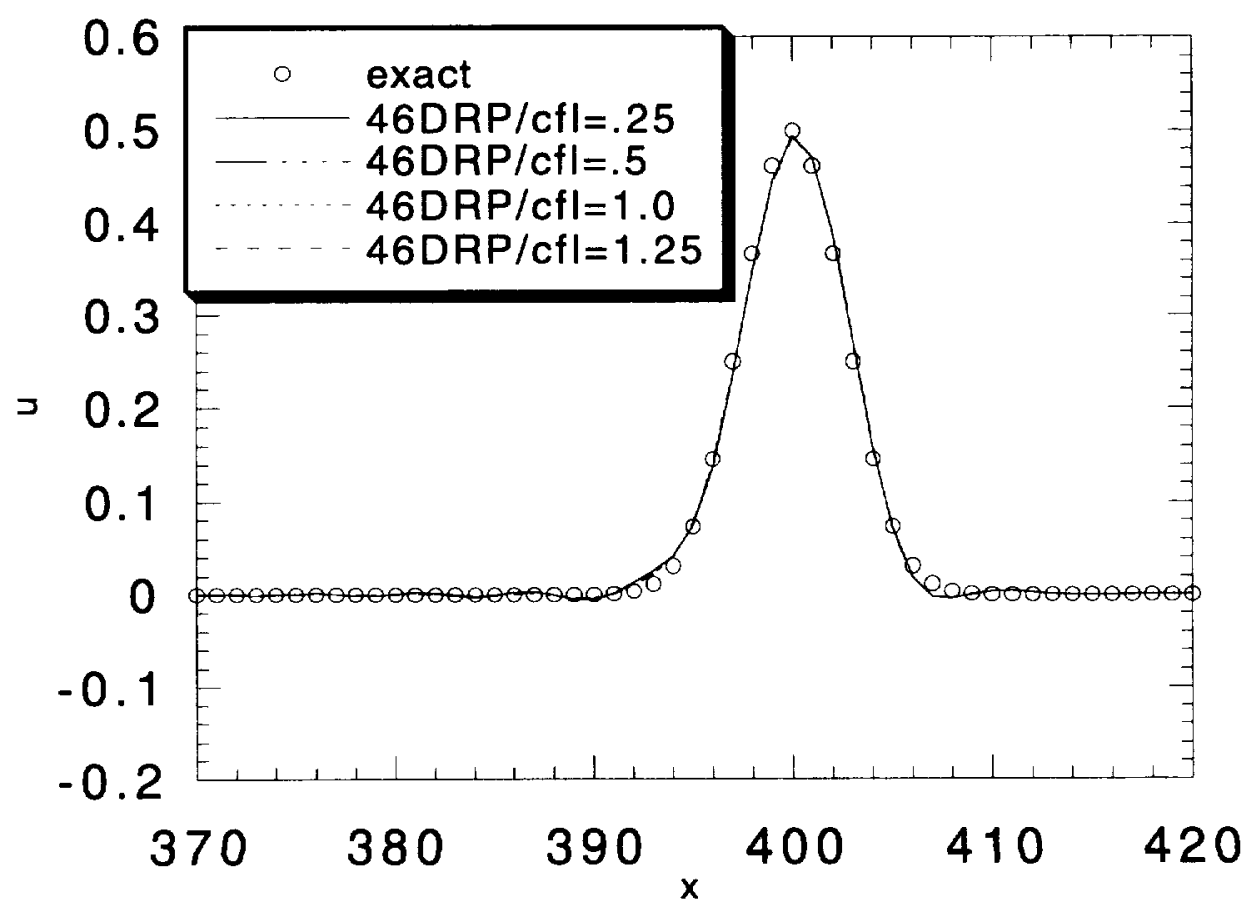

Figure 5.-Solution of Benchmark Problem 1 of the First CAA Workshop using Optimized DRP Scheme. 


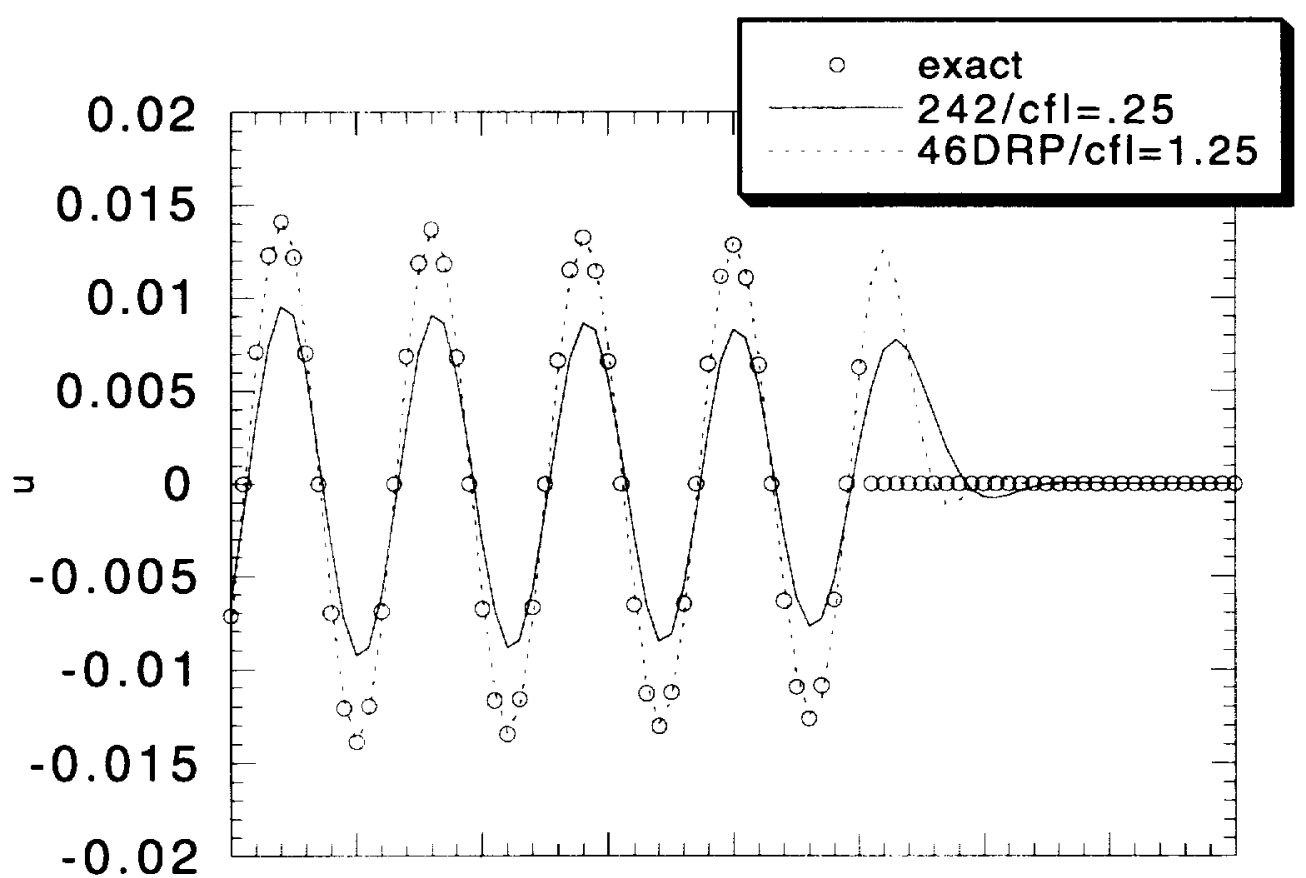

350360370380390400410420430

Figure 6.-Comparison of Solutions of Benchmark Problem 2 (12 points/wavelength).

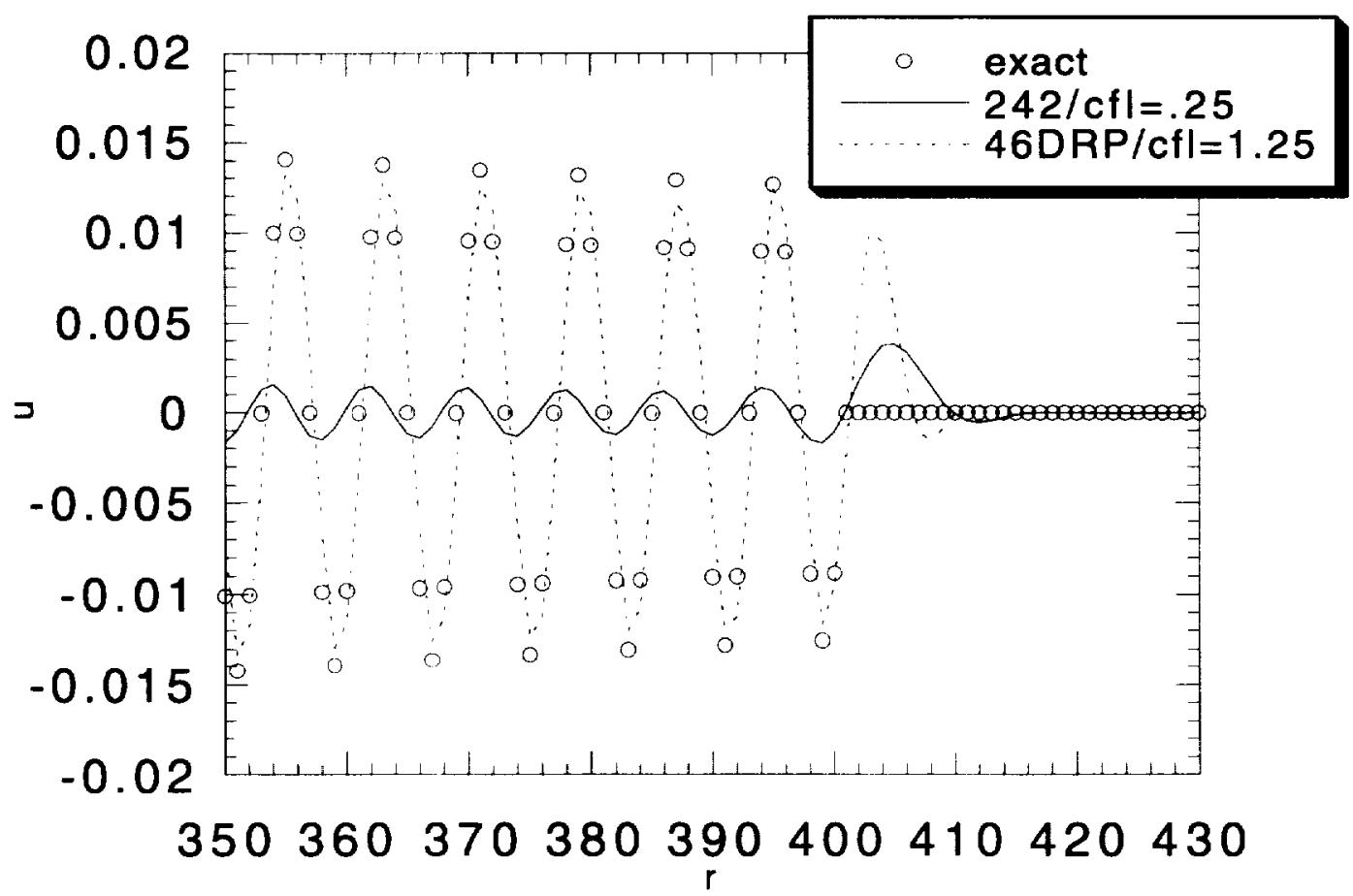

Figure 7.-Comparison of Solutions of Benchmark Problem 2 (8 points/wavelength). 


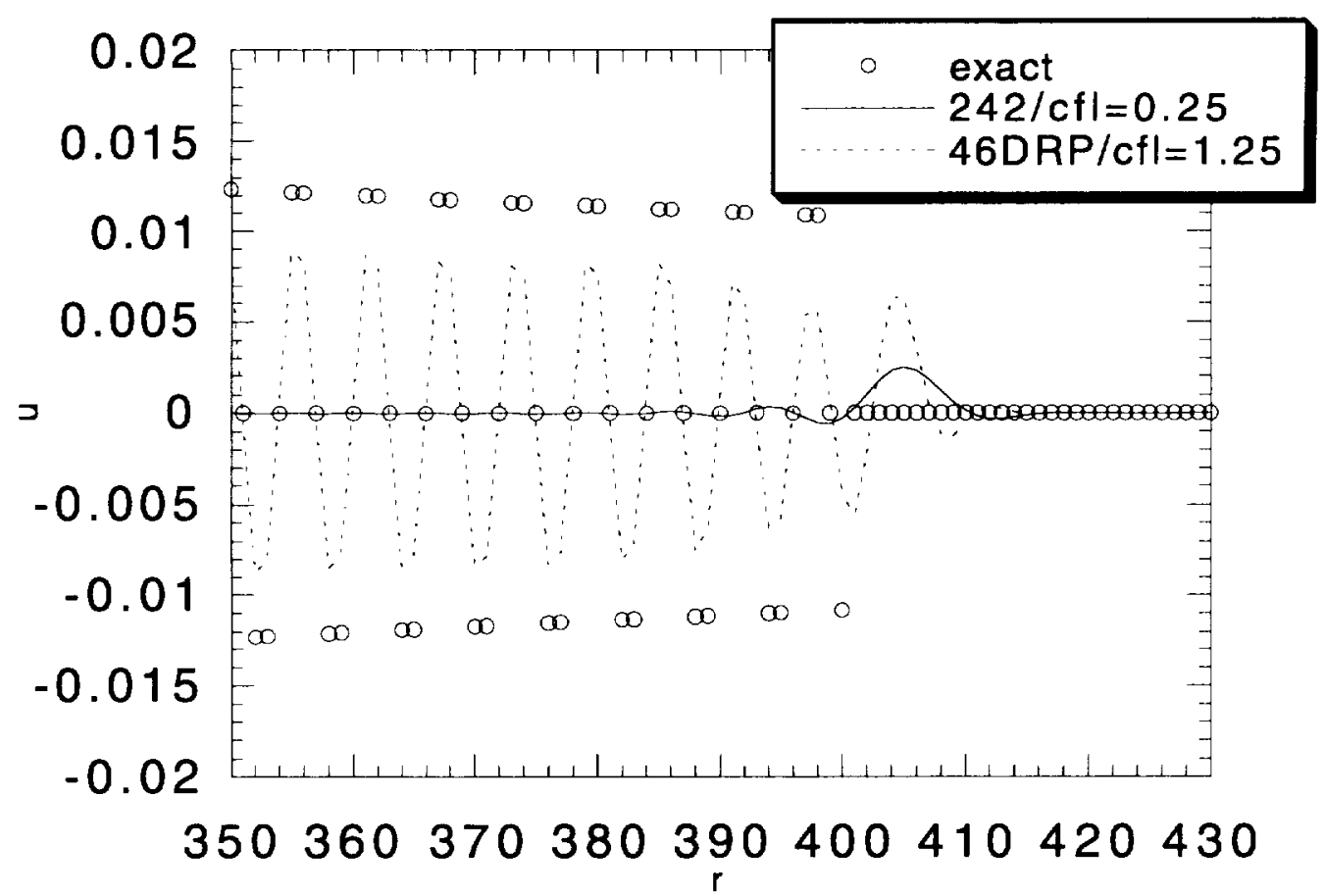

Figure 8.-Comparison of Solutions of Benchmark Problem 2 ( 6 points/wavelength).

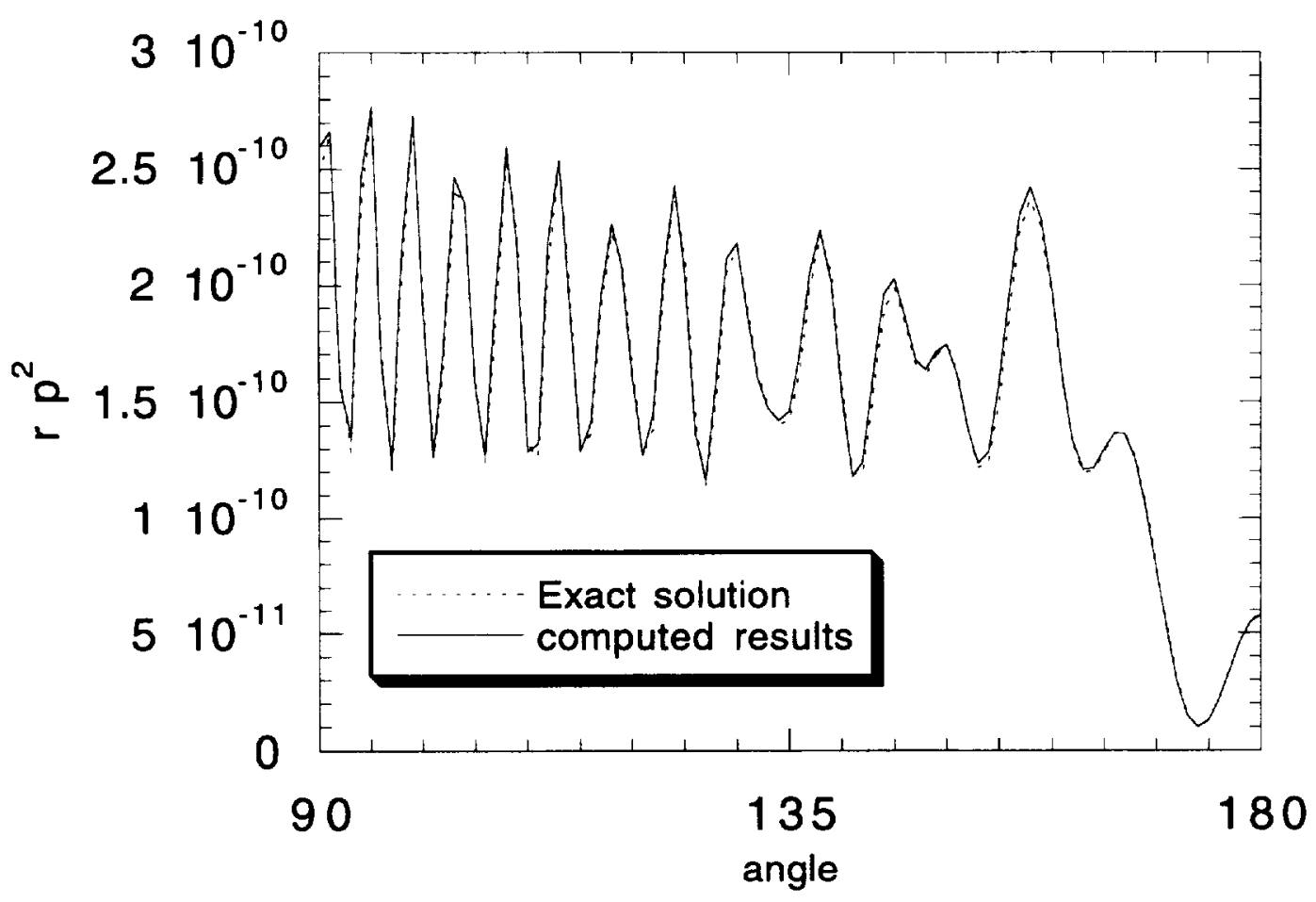

Figure 9.-Computed solution of Problem 1 of the Second CAA Workshop at $r=15$ using Optimized DRP Scheme. 


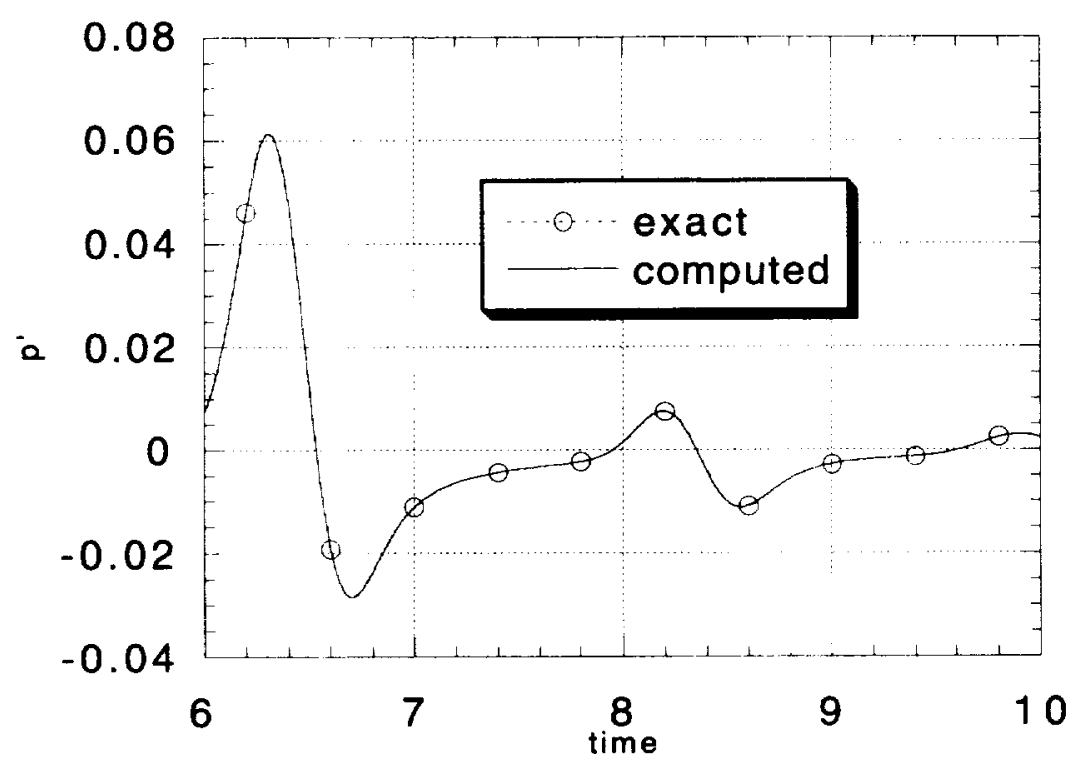

Figure 10.-Computed solution of Problem 2 of the Second CAA Workshop at point A using Optimized DRP Scheme.

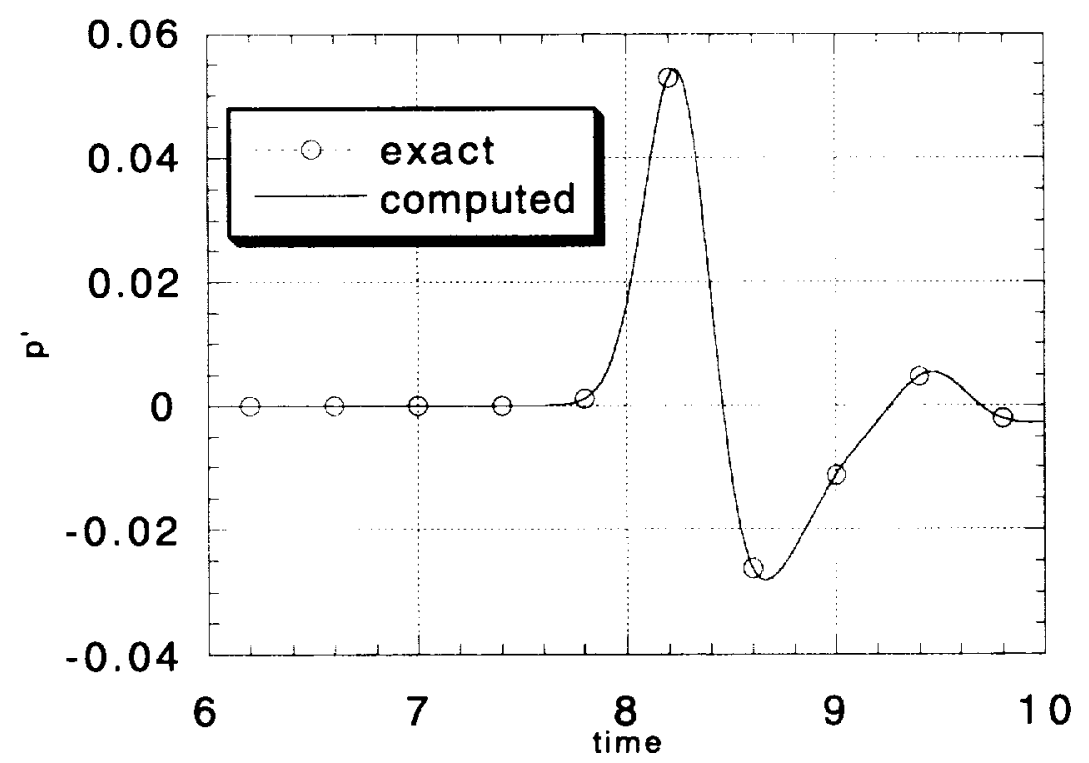

Figure 11.-Computed solution of Problem 2 of the Second CAA Workshop at point B using Optimized DRP Scheme. 


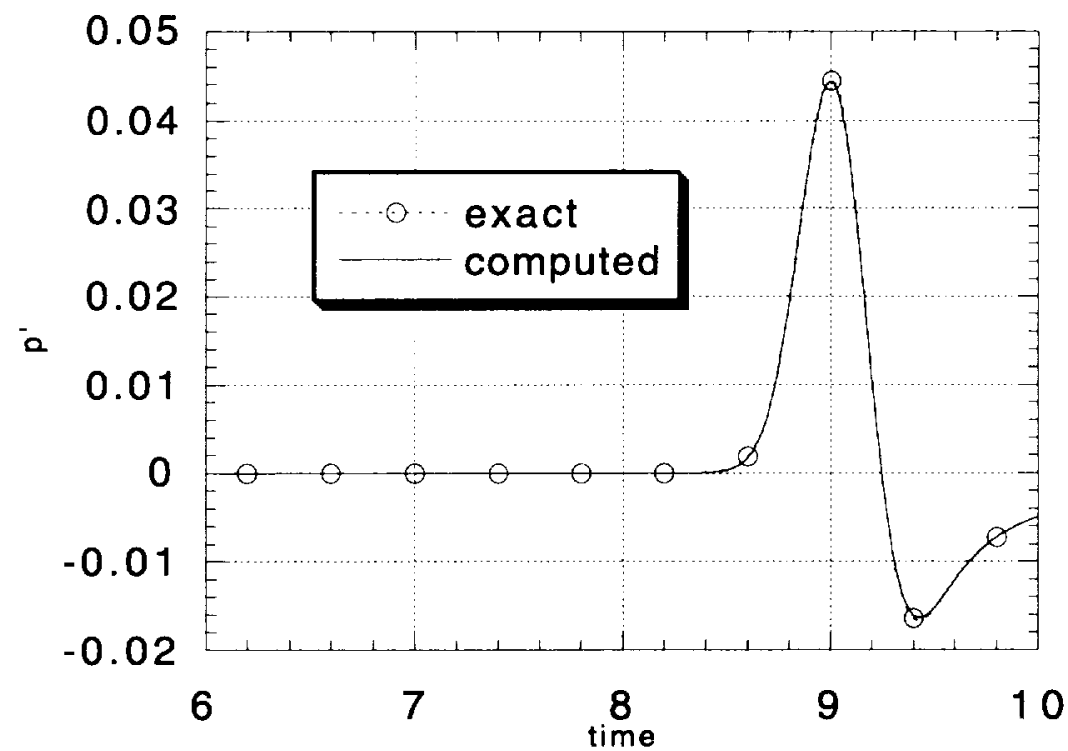

Figure 12.-Computed solution of Problem 2 of the Second CAA Workshop at point C using Optimized DRP Scheme.

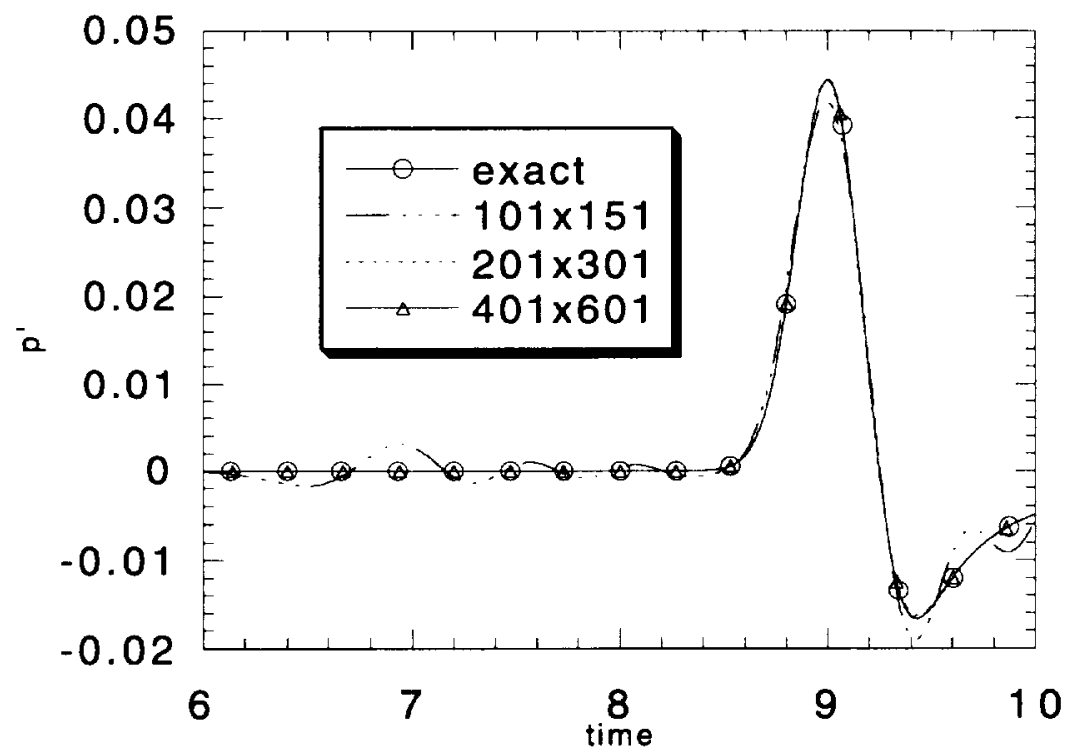

Figure 13.-Effect of grid density on computed solution at point $\mathrm{C}$. 


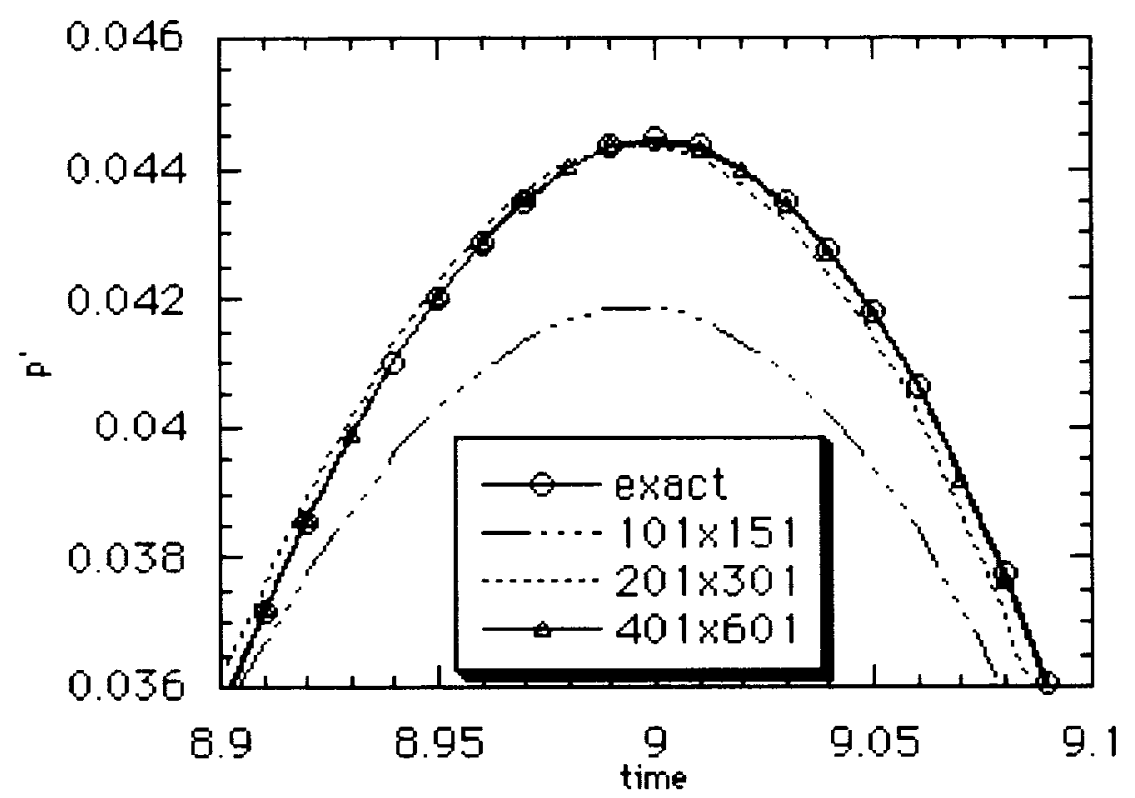

Figure 14.-Effect of grid density on computed solution for transient peak at point $C$. 

Public reporting burden for this collection of information is estimated to average 1 hour per response, including the time for reviewing instructions, searching existing data sources, gathering and maintaining the data needed, and completing and reviewing the collection of information. Send comments regarding this burden estimate or any other aspect of this collection of information, inciuding suggestions for reducing this burden, to Washington Headquarters Services, Directorate for Intormation Operations and Peports. 1215 Jefferson Davis Highway, Surte 1204, Arlington, VA 22202-4302, and to the Office of Management and Budget, Paperwork Peduction Project (0704-0188), Washington, DC 20503.

\begin{tabular}{|l|l|l|}
\hline 1. AGENCY USE ONLY (Leave blank) & $\begin{array}{c}\text { 2. REPORT DATE } \\
\text { March } 1997\end{array}$ & $\begin{array}{r}\text { 3. REPORT TYPE AND DATES COVERED } \\
\text { Contractor Report }\end{array}$
\end{tabular}

\section{TITLE AND SUBTITLE \\ Evaluation of a High-Accuracy MacCormack-Type Scheme Using Benchmark Problems}

6. AUTHOR(S)

R. Hixon
5. FUNDING NUMBERS

WU-523-36-13

NCC3-531

8. Performing organization REPORT NUMBER

E-10656

22800 Cedar Point Road

Cleveland, Ohio 44142

10. SPONSORINGMONTORING AGENCY REPORT NUMBER

National Aeronautics and Space Administration

Lewis Research Center

Cleveland, Ohio 44135-3191

NASA CR-202324

ICOMP-97-03

11. SUPPLEMENTARY NOTES

ICOMP Program Director, Louis A. Povinelli, organization code 5000, (216) 433-5818.

12. DISTRIBUTION/AVAILABILITY STATEMENT

12b. DISTRIBUTION CODE

Unclassified - Unlimited

Subject Category 64

This publication is available from the NASA Center for AeroSpace Information, (301) 621-0390.

13. ABSTRACT (Maximum 200 words)

Due to their inherent dissipation and stability, the MacCormack scheme and its variants have been widely used in the computation of unsteady flow and acoustic problems. However, these schemes require many points per wavelength in order to propagate waves with a reasonable amount of accuracy. In this work, the linear wave propagation characteristics of MacCormack-type schemes are shown by solving several of the CAA Benchmark Problems.

14. SUBJECT TERMS

Computational aeroacoustics; MacCormack scheme; Benchmark problems

15. NUMBER OF PAGES 24

16. PRICE CODE

$\mathrm{A03}$

\begin{tabular}{|c|c|}
\hline $\begin{array}{c}\text { 17. SECURITY CLASSIFICATION } \\
\text { OF REPORT } \\
\text { Unclassified }\end{array}$ & $\begin{array}{c}\text { 18. SECURTY CLASSIFICATION } \\
\text { OF THIS PAGE } \\
\text { Unclassified }\end{array}$ \\
\hline
\end{tabular}
19. SECUAITY CLASSIFICATION OF ABSTRACT

20. LIMITATION OF ABSTRACT

NSN 7540-01-280-5500 